Hospital Luis Calvo Mackenna.

Cátedra Extraordigaria de Pcdiatría

Prof. Anibal Ariztia.

\title{
TRATAMIENTO DE LA MENINGITIS TUBERCULOSA CON RSTREPTOMICLNA
}

IComunicación preliminar

\author{
Por los Dres, OSCAR llLANES BenItez \\ y MARIANO LATORRE BLANCO
}

Esta comunicación preliminar tiene por objeto contribuir con nuestra experiencia sobre los resultados obtenidos en el tratamiento de la meningitis tuberculosa con estreptomicina.

Cen miras a este propósito presentamos la evolución clínica de dos pacientes con meningitis tuberculosa, actualmente en tratamento con esta drega, en los cuales hemos podido confirmar el diagnóstico con la identificación del bacilo de Koch en la inoculación experimental y cultivo del líquido céfalo raquídeo obtenido de la primera punción diagnóstica.

De estos dos pacientes, uno se encuentra hospitalizado en el Servicio de Pediatria de la Cátedta Extraorfinaria de Pediatria del Prof. Ariztia del Hospital Luis Calvo Mackenna. El otro pertenece a la clientela privada, atendidos por perscinal médico y labcratoristas de este mismo servicio.

Si bien nuestra reciente experiencia no nos permite asegurar que estos pacientes puedan llegar a obtener una mejoría clínica definitiva. podemos afirmar que el resultado inmediato de la estreptomicina es sorprendente, al producir una rápida regresión de los signos clínicos de la meningitis tuberculosa.

Este solo heche nos permite valorar la importancia trascendental del descubrimiento de la estreptomicina por los in- 
vestigadores americanos Schatz. Bugie y Waksman, que abre un nuevo horizonte, lleno de promisoras esperanzas en la terapia de algunas fotmas de complicación de la tuberculosis y en el importante rubro de las enfermedades producidas por gérmenes gram negativos, para los que las nuevas drogas con que cuenta la terapéutica moderna han tesultado de escasa o ninguna eficacia.

No es nuestro propósito entrat en consideraciones sobre Ics resultados obtenidos con esta terapia en otros procesos patológicos o en otras complicaciones de la tuberculosis. Sin embargo. consideramos de vivo interés hacer una breve reseña de los casos de meningitis tuberculosa tratados con estreptomicina y publicades en la literatura médica durante este último tiempo: ia mayoría de los cuales pertenece al equipo médico que ferman el National Research Council. dirigido por Chester S. Keefer, encatgado de las experiencias preliminares en diversos procesos patológicos y de $\mathrm{H}$. Corwin Hinshaw y William H. Feldman, que se hicieron cargo de las investigaciones en tuberculosis y que incluye varios casos de meningitis.

Louis L. Krafchick, en el J. A. M. A., del 19 de octubre de 1946, relata un caso de un lactante de 15 meses con sintomas clínicos de meningitis tuberculosa y tuberculinas positivas, que fué tratado con estreptomicina durante 57 días: con un total de 24 grs. intramusculares y 2 grs. 800 malgrs. intrarraquideos y un tetal de 65 punciones lumbares. El líquido céfalo raquídeo mostró, en una ocación, bacilos ácido resistentes, sin embargo, el cultivo fué siempre negativo $y$ también la incculación experimental.

Vale la pena consignar que en el curso de la enfermedad se produjo una parctiditis con fuente de contagio familiat.

El liquido céfalo raquídeo demostró pleocitosis moderada. 5 meses después de inciada la enfermedad.

En el relato del National Research Council se menciona un caso publicado por Cooke, Dunphy y Blake en el Yale Journal of Biology and Medicine de 1946. de un paciente de 1 año de edad con curación clínica y bacteriológica de su meningitis tuberculosa.

Nuestra experiencia se refiere a dos casos tratados con estrepromicina: una niña de 13 años, cuya enfermedad se inicia hace 7 meses, y un lactante de 2 años. que inició su enfermedad hace 3 y medio meses. 


\section{OBSERVACION $N: 1$.}

Chica de 13 años, cuyos antecedentes mórbidos no tienen importancia. No se ba podido establecer conlagio tuberculoso intra lamiliar; sin embargo, es ncesario tousignat el hecho que una tía $y$ un abuelo faliecieron de un proceso tuberculoso. Antes de la enfermedad actual no habia evidencia clinica ni radiológica de haber padecido de un proceso tuberculoso.

Enfermiedad actual. - - Se inicia el 12 de ntoviembre de 1946 con pequeñas cefaleas. Posteriormente se establect un cuadro febril discreto, con temperaturas de tipo remitente, acompañado simpre de cefaleas cada vez más intertsas. a la cual se agrega constipación. En estas condiciones se mantiene 13 dias, al cabo de los cuales la cefalea se hace may incensa, apateces vomitos incoercibles, que hacen presumir una meningitis tuberculosa, que to confirmada por punción lumbar. Al día siguienle aparecen lus signos característicos del compromiso meningo encefalico: rigidez de nuca, Kernig y Brudsingky de media nd intensiad. anisocoria con reacción pupilat floja a dercha, dermografistro inuy acestuado, estado de semi inconciencia.

Vicciseis dias despues se inicia terapia con estreptomicina exclusivament por via intraraquidea, coincidiendo con un estado de franca anravación y acenruación manificsta de los sintonas ya mencionados en forma que se hace prás. ticamente impostble lo alinentacion. Las primeras dosis si inyectaron exclusivamente por vía intraraquidea a razón ds I gr. en 2 ingecciones. sepatadas por 12 horas. Esta desificación se mantuvo durante 4 dias, al final de los cuales. debido a la escasce de troga. so haja a $300 \mathrm{mlg}$ s. diarios, que st mantienen hasta el décimo quinto dia.

Durante los 3 primeros dias no se nota mejorid clinica ni subjetiva. antes bien. el dia 30 (18 dias de iniciada la enfermedud y 3 de iniciado el Iratamientol se observa una agravación. que nos hiro prever muy poias esperanzas respecto al éxito. En esta ocasión, Ja tinferma estaba co coma: compro. miso de los pares cenneanos en forma de una ptosis deceha con midriasis. Fenómenos babures (ctisis de aprea muy prolongadas $y$ cianosis con puiso irregular). Al da siguiente y con sorpresa se nota mejorid, que consiste en racuperación parcial del sensorio (contesta preguntas y expresa sus descos). En los dirs siguientes se va acentuando paubtinamente la metoria $y$ baja la temperatura. que se habia mantenido entes 38 y $39^{\circ}$. Al mismo tiempo a usa sintomas de compromiso del octaro par (zumbidos de oidn, ruidess extraños. hipoacusia. mareo y vómitos). Al sexto dia de iniciado el tratumienio hay som. pieta recuperaición de la lucidiez. La enferma conversa, se alimenci er buenas condriones y es capar. de sentarse. Los simtomas meningeos ban disninuido notablemente; pero persiste cierto grado de rigidez de nuca, que no guarda rilacion con ta recuperación franca de los sintomas subjecivos. Como signos inter. carrentes aparece prurito inténso de las extrimidades. y región perícal: citmentos de miero pápulas en la cara y miembros. Ademis. elementos purjucicos de pequeño camsäo en la nutión interna del firazo derecho $y$ manchas rojas 
en nape en anbas mejillas. En reizción con la punción lumbar se manifirstan intensos dolores radiculates y trastornos csfinterianos.

A los 15 dias de iniciado el tratamiento, se produce una acentuación de fos signos meningeos; aumento de la temperaura con desmejoría de' estado general. Este fenómeto fuć interpretado como una agravación por dosis insuficientc, que nos habiamos visto forzados a disminuit en atención a la poa disponitbritidad. Esta circunstancia nos bizo reforzar la cantidad de fruga intrateial cesde el 15" a! 22" dia, en cantidades yue fiuctuaron entre l y 1.5 gr., dir:didos en 3 inyecciones intraraquideas diarias.

En relación con esti aumento se estableció und rigidzz incensa de la nuca y di las extremidades. basta llegar a la jrosición de opistolonos, junto a un sopor intenso semejante al observado in cierias tipas de cniefalitis, paes la inferma renia cortos periodos de lucidez, para luego caer en sopor. La obsevación atesla de este feriomeno nos hizo diferenciarlo del verdadero coma ancningeo por la conservazion completa de su psiquismo durante los intervalos de lacidiz. Todo esto. aunado al liquida cúfalo raquideo, cuyo examen ros dio clorueas y gincosa normales con aumento considerable de la dibumina y discre10 de los elementers igurado3. nos permilio sopkechar yuz este complejo sincomático juesa dabido más bien a la acción de la droga. Jin presuncia de este cuento procedimos a suspender b medicacion por 24 horas, al cabo de las cuates obtuvimos regresion maniliessa de hos sintomas ya descritos.

Se reinicia el leatamento a los 23 dias adoplando ba ria combinada inItatecal e intramuscular, gue permitio inyectar dosis minotes co el canal, manceniendo da dosis sotal diaria en l gr., diviclido en 900 mlgrs. intramuscular y 10 mogrs. intraraguideo. En el mes siguiente se mantiene esta dosis y se tonccden costos prtiodos de discinso y variationes en el ritmo de las punciones lumbars. de acuerdo con la mejor a clinica de la enfermila. A los 2 meses de iniciato at craramiento. se suspende la droga por 2 meses. En al interwalo se hoce una punción lumbal de inutrol. que reveló normulidad en tas cifras de c'orures y gluross, alln alando existia todavín un cierto grado de procinous con discreta atbuminosis.

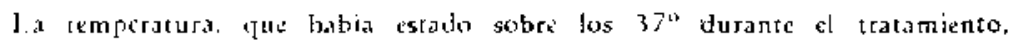
se normatiza a los 0 stius de disiansu. El estado geniral es toreciente. Sube de jeso. Juega $y$ lee y ensaya sus primeros pasos. No guarda alma. Se peactica lamber en iste intervalo und tadiografia pulmonar. que revela: "Sombras estompadas muy teaues de iniliración en región apica! y sub clavicuiar derechas y tercio mediu izquirdo. Prefueñas vatilicaciones en of $1 / 3$ medio de ambos campos pulmonares".

Et 13 de febrero se autorsia et suy inoculado y se constata disaminación tuberculosa. Protocolo dice:

I.. C. R. Inoculación experimental practicadd el 7 de dicimbre de 1.946.

Dos cuyes autersiados a los 2 y medio meses de la inoculación prescotan tuberculosis generalizada. 
Uno de ellos, inoculado subcutíneamente en la región abdominal, presenla un ganglio vecino al punto de inoculación, en franco proseso de caseifi. cación. Se comprueban tubcrculos ea los pu!nones, higado y bazo.

Ef segundo cay inoculdo intraperitoneamente, paejenta una masa fibco. caseosa en el epiplón. St comprueban tubírculos en tos pulmones, higado y bazo, y la presencia de barilos de Koch en ellos.

15 de febrero del 47.

(Fdo.): Dr. Emilio Prado.

\section{OBSERVACION N' 2 .}

Lactante de 1 año 10 meses, que ingresa a la sàia de Neurologia del Sur. ricio de Recepción del Hospital l. his Calvo Mackental con una enäermedal accual que se inicia 10 dias atrís, con malestar general y fiebre discrua. Luego rómitos frecuentes de arácter fícil $y$ explosiros. A pesar de los tratamientos instituidos persiste la sintomatologia. a la que se agrega somn:Iencia y enbotamiento del sensotio. Constifación de hasta 2 dias. Mantoux practizado en esta ozasión resulta intensamenle positivo. Sedimentación de lj mm. Radioszopia aue jníorma "sombra ds infillacion pulmonar derecha".

En su domicilio es vista por varios médicos. que constatan la presencia de signos meningeos y solicitan punción lumbar. cuyo resultado es el siguiente: albúmina, 0.70 gr.; clorufos. 6.90 grs.: glucosa, 0.35 gt.: Fandy y Nonn: +++ Wass. (-). Formula, $160 \mathrm{x} \mathrm{m}, 3$. son $98 \mathrm{k}$ linfocitos y $2 \mathrm{r}$ monotitos.

Antecedentes familiares. -- Madre sutrió lesion pulmonar. Alta ha:a 7 años.

Antecedentes personales. -- Concagio con tuluzculosis abizts intrafamiliat.

Ingresa el 22 de marzo con 11 kgrs, y a sn ingreso se constata un buin estado nutritice. Decaimierto. Sensorio algo embotado (bosteza constant: mente). Discreta hipotonis muscular Intensa rigidez de nuca. Kerrig y Bruf. zinsky intensamente positivos. Ingresa con el diagnostico de complejo primario derecho en evolución. Seningitis tubeculos.1.

Esa misina turde sc inicia tratamiento con estreptomicina: 100 migrs. dia. fios intratecales y 150 m!grs. cada 4 horas por via intramuscular. completctado 1 gr. diario por anbas vias. I.a prioers muestra se utiliza para siembea e inoculaciós expetinentá, Durante 0 dias se mantuvo este ratamiento. Los signos meningros anotan un regreso recién al $7^{\circ}$ dia de instituidos, previo un corto periodo de hipetrigide. El apetito, sin embargo. mejora y el riño apt rece más animado.

Al 10 " dis se froduce el primer sintoma de intoxicación. bue consistió en nislagmus $y$ molestia vaga. que hizo lorar al lactante durante 24 horas sir: salusa aparente. Pot csta razon se bain la dosis intratecal a 50 migrs. diation. manteniendo la intramuscular en $900 \mathrm{~m}$ ggrs. Una radiografía de tórax tomada en ess fecha mbesira su compleịo primario sin mayor variación. 
La nueva dosificación se mantient hasta al $15 \%$ dia. en que nuevos signus de intoxicación. que consistiefon cn alza térmica a $39.8^{\circ}$. convulsiones y gran entotamiento sensorial, obligan a una nueva disminución de la dosis. iniciác:duse el siguince esquema: 600 mlgts. diarios por via intranuscular y 50 migrs. intratecales dia por medio. Rapidamenta se obsetva un descenso de la temperatura en lisis y la mejoria clinica st hace mis $y$ más svidente. Desaparecen los signos meningeos al 25" día de tratamiento. establaciéndeiz una enejurid un tlara, que el niño no pátece enfermo. la tempecatuta se manciene baja. co: alyas periódicas en relación con la inyeción intrutecal. Se decicie espaciarlas 3 colda 2 días y luego a cada 3 dias. La mejoria clinica cbtenida st consolid. en forma tal. que el niño. an la actanidad pucde considerarse normal para su edad.

Dinarte el curso d: la heopitalización, se ha pracricado ua control ta diografico quincenai, con ef fin di observar ios cambios que pudieran producirst. Las radiografias no han mostrado hosta la fecha ningún cambio en la imıgen de su complejo primario. La sedimencación practicada a intervalos if mas o menos 25 dias. Se mantuvo invarialule alradedor de $20 \mathrm{~mm}$. para loig", desecnder a 8 y $6 \mathrm{~mm}$. por hora ca el último control practicado.

Un mes y 7 dias de haber ingresado, el cultivo de bacilos de Kais resulta positivo con lo que sé confirma bacteriológicamenta el diagnóstico.

\section{Técnica de tratamiento.}

Hemos querido agrupar bajo este nombre todo lo que se refiere a la dosificación y técnica de introducción de la droga.

1) Aunque parezca superfluo insistir en la perfecta realización de la punción lumbar. creemos vale la pena, porque siendo éste un tratamiento largc. mayor que cualquiera de los actualmente conocidos. la perfecta técnica de la punción permire utilizar en forma casi indefinida el canal raquídeo. Es necesaric recordat que diversos factores influyen para alterar las condiciones del canal. Ellos sen: el drenaje continuc, la inyección de materias cxtrañas de suyo irritantes y la punción mal efectuada. Si pensamos que los dos primeros factores están siempre presentes, se ve la necesidad de obviar el tercer factor. que es de exclusiva dependencia del médico.

2) Un segundo punto que merece considerarse es la dilución y el tipo de diluyente a usar en la inyección intratecal. De la experiencia adquirida (que alcanza a más o menos 200 inyecciones intratecales de esta sustancia), hemos podido deducir algunos hechos de interés. $\mathrm{La}$ dilución ha de hacerse en la cantidad máxima pasible, por cuanto la droga. con su afinidad neurotóxica, provoca violentas reacciones. 
que se han traducrdo en dolores raquiálgicos inmediatos, que llegan a hacerse intolerables, impidiendo la prosecución del tratamiento. Esta circunstancia ha podido ser sorteada mediante la dilución extrema de la droga en el momento de ser inyectada. Dilución que varia según la cantidad usada; pero que puede generalizarse en más o menos $5 \mathrm{cc}$. por cada 100 mlgrs. de droga.

El tipo de diluyente influye también en la atenuación de los fenómencs dclorosos ya mencionados y en términos generales puede decirse que el diluyente ideal es el propio hiquido céfalo raquídeo. Se consigue asi una más perfecta esterilidad, un mínimo de manipuleo y un máximo de tolerancia.

Vale la pena consignar que dentro de este capitulo es necesario insictir sobre un hecho que de momento parece de poca importancia, pero que posteriormente es de absoluta necesidad y es que la primera muestra obtenida ante la posibilidad de iniciar tratamiento debe utilizarse para siembra e inoculación experimental, ya que muestras posteriores no permiten cl desarrollo del bacilo de Koch por la acción inhibidora de la droga. Es también necesario un control semanal químico y citológico, que permite variar la dosificación y periodicidad del tratamierito.

Hay un hecho digno de mención en el tratamiento de nuestros cascs. En la Obs. $N^{\circ} 1$ se puede observar que el tratamiento inicial fué intratecal exclusivo. La razón es simple y fué principalmente debido a nuestra ignorancia en el manejo de la droga, pues no habia hasta entonces experiencias preliminares. En segundo término, la gravedad del caso nos indicó como vía principal de ataque la intratecal exclusiva, máxime cuando a este se unía una cantidad exigua de droga, que nos hizo reflexionar si no setía un desperdicio el utilizarla por via intramuscular.

Respecto a la dosificación de la estreptomicina hay hechos previos que considerar: se ha probado que el uso demasiado cauteloso de este medicamento es capaz de producir con esta droga más que con ninguna otra la resistencia del germen. Por esta razón, y en términos generales, podemos decir que su dosificación la hemos efectuado siguiendo una pauta un tanto extraña. por cuanto hemos exagerado la dosis, manteniéndo1a en el límite máximo de tolerancia, es decir, bcrdeando la intoxicación y así se puede ver en los casos expuestos que. lactantes de peso y talla totalmente diferentes, han recibido una cantidad igual o apenas menor que la de un adulto. En esta dosificación limite juega un rol preponderante la observa- 
ción y valoración clínica de los síntomas de intoxicación. Por ello, en aquellos enfermos (Obs. 2) en que la sintomatclogía subjetiva es nula, hemos procedido a observar los signos clínicos acusados por el octavo par y darles alcance de índice terapéutico efectivo. En las observaciones recién mencionadas hubo nistagmus espontáneo, al que se agregó estrabismo convergente en la Obs. $N^{\circ} 1$ y ataque convulsivo franco en la $N^{0} 2$. Estos hechos, unidos a malestar general y alza térmica abrupta, nos marcaron el límite de la tolerancia y por ende la baja del dosaje por ambas vias. La observación clínica posterior nos permitió mantener esta nueva dosis o aun bajarla; perc ahora. de acuerdo a indices de mayor cstabilidad y valor clínico, como fueron la temperatura, mejoría clínica y liquido céfato raquídeo.

En lo que respecta a la temperatura. es necesario precisar que ésta se mantiene sobre 37 mientras dura el tratamiento, por lo tanto, es de un valor relativo en cuanto a indice de mejoría clínica. En nuestras dos observaciones hemas podido verificar que ésta se hace normal una semana después de haber suspendido totalmente el medicamento. Es de interés hacer notar que en las sucesivas punciones se producen violentas alzas, hasta 41\%, en estricta relación con ellas (Obs. ] y 2 ).

El liquido céfalo taquídeo sigue un curso algo más prolongado que los dos índices anteriores y se observa que las cifras de glucosa y cloruros muestran tendencia al aumento al cabo de más o menos 15 días, en tanto que la albúmina y elementos aumentan en forma tal, que hacen creer en la agravación del cuadro meníngeo, cuando en realidad no es sino el retrato de la irritación difusa provocada por el medicamento en el canal raquídeo. La normalización relativa del líquido céfalo raquídeo sólo se obtiene un mes después de haber suspendido totalmente el medicamento.

Los períodos de descanso los hemos subordinado a estos tres factores. es decir: mejoría clinica. baja de la temperatura y normalización relativa del líquido céfalo taquídeo.

En nuestras dos observaciones el periodo inicial de tratamiento ha durado más o menos 2 meses y en estos 2 meses hemos obtenido la mejoría clínica completa del cuadro meníngeo, la baja de la temperatura con alzas periódicas en relación con la inyección intratecal (Obs. 1 y 2) y la normalización relativa del líquido céfalo raquideo. En este descanso total del medicamento bemos hecho control semanal del líquido céfalo raquideo, que aparte de darnos una visión del 
estado meníngeo, junto a los otres indices. nos pernite reiniciar el tratamiento antes que se produzca el cuadro completo.

En lo que respecta a la vía intramuscular, es necesario tener en cuenta que la inyección de por sí dolorosa, se hace casi intolerable a medida que transcurren las semanas, 11 egando a producirse infiltraciones de músculos que impiden la mevilidad espontánea y que contribuyen a dar esa especie da paresia que los enfermos presentan en 'el curso del tratamiento. Esta dificultad puede obviarse en parte, mediante el agregado de $1 \mathrm{cc}$. de solución de procaina al $1 \%$ por cada $4 \mathrm{cc}$. de solución de estreptomicina. En tedo caso, en quellos enfermos (Obs. 1 y 2) que han tenido un descanso prolongado en el curso del tratamiento. se ba observado la rápida mejoría de estas alteraciones.

\section{Fenómenos intercurrentes en el eurso del tratamiento.}

Nos parece de interés consignar en un párrafo aparte los fenómenos intercurrentes que hemos observado en el curso del rratamiento de la meningitis tuberculosa con estreptomicina.

Estcs fenómenos se refieren a efectos neurotóxicos de $1 \mathrm{a}$ drcga sobre el sistema meningo encefálicc. nervios craneanos. filetes sensitivos de la cola de caballo y manifestaciones qu: corresponden a las reacciones histamínicas generales.

En las dos observaciones hemos visto, con mayor a menor intensidad que, durante el curso del tratamiento con inyección diaria de estreptomicina intratecal. se produce uri estado de hiper-rigidez de los mísculos de la nuca hasta llegas a la posición en cpistótonos e incluso hipertonía de los miembros superiores e infertores (Obs. $\left.\mathrm{N}^{9} \mathrm{i}\right)$.

Aparentemente esta condición sugiere una agravación de los signos meníngeos; pero un hecho curioso que nos llamo la atención, es la manifiesta discordancia que existía entre la intensidad de la rigidez y la completa lucidez mental, junto a la regresión de la temperatura y orros signos meníngeos. Por otra parte, el líquido céfalo raquideo reveló la presencia de gran cantidad de albúmina con aumento de los elementos figurados: en tanto que la glucosa y los cloruros mostraron cifras casi normales. La consideración de estos hechos nos hizo pensar que los fenómenos de biper-rigidez y las alteraciones anctadas en el líquido céfalo raquídeo serían condicionadas por la misma drega. En efecto, con la reducción de la dosis o con la supresión de las inyecciones intraraquí. deas por uno o dos días, observamos una regresión franca. 
En la observación $N^{\circ}$ l anotamos un Fenómeno que, a nuestro parecer, ha sido un compromiso encefálico, pues se caracterizó esencialmente por tendencia muy definida al sueño profundo, casi soporoso durante 48 horas, con conservación completa de la lucidez mental y sin la intercurrencia do ningún otro signo meníngeo. La chica permanecia en un sueño profundo, tranquilo; al despertarla, contestaba con plena conciencia y con un perfecto dominio de sus facultades mentales. En un principic pensamos en la agravación del cuadro: pero, luego, la observación atenta nos permitió apreciar que el estado de sopor no tenía carácter meníngeo e inculpamos a la droga, procediendo entonces a interrumpirla por 24 heras, al final de las cuales abtuvimos una vuelta a la normalidad.

La acción selectiva de la estreptomicina por el VIII par se puse en evidencia en un case (Obs. $N^{\circ} 1$ ). Como síntomas frodrómicos pudimos anotar: ruidos extraños, zumbido de cidos, hipoacusia y luego sordera completa. También se evidenciaron síntomas de compromiso laberintico, como vértigo. ráuseas y vómitos, que se ponian de manifiesto cuando la enfermita se incerporaba en la cama.

En una casión observamos, a raiz de la invección intratecal, ligero grado de estrabismo y diplopia. que duró algunas horas (Obs. No 1). En el otro caso (Obs. $\left.N^{\circ} 2\right)$, a los 15 días de iniciado el tratamiento, se produjo, como único sintoma cbjetive de compromiso vestibular, un nistagmus persistente con desviación lateral de más o menos una hora de duración.

Los dolores radiculares que se producen con la inyección intratecal de la droga no serian absolutamente imputabies a la acción neurotóxica de la estreptomicina, porque también lc hemcs podido observat con las inyecciones prolongadas de penicilina intratecal. En consecuencia. es posible que en este caso la droga actúe sólo como sustancia extraña en el canal raquideo.

Otros sintomas que serian atribuibles al compromiso de las fibras censitivas de la cola de caballo. es cierto grado de paresia que se cbserva en el intestino y vejiga con inhibición del reflejo de la defecación y mícción. al parecer de carácter reversible. Estas alteraciones obligan a menudo. cuando no se produce micción por rebalsamiento, a vaciar la vejiga por compresión de la región suprapubiana. Para la evacuación del intestino hemos procedido a administrar gran cantidad de 
Eustancias que obran por acción mecánica de arrastre o al sifonaje intestinal.

Entre los sintomas neurotóxicos observados durante $a$ tratamiente, hemos visto 1 Obs. $N^{\circ} 1$ y en otro no incluido en esta comunicación) que al comienzo del tratamiento se preduce un pronunciado grado de insensibilidad. principalmente de las extremidades inferiores, que puede o no ser simétrica, con conservación de los movimientos de defensa.

Entre las manifestaciones que corresponden a las reacciones histamínicas generales, debemes mencionar. en primer término, por el gran malestar que provoca al enfermo, el prurito generalizado, de preferencia anovulvar y cuya intensidad llega a provocar crisis de excitación psico motora e insomnic. Este fenómeno, condicionado por la alta dosificación. no cede a ningún tratamiento habitual, salvo la supresión o disminución de la estreptomicina.

Dentro de este mismo grupo cabe mencionar la aparición d. blementos micro papulosos. que se establecen de preferencia en la cara de extensión de las extremidades: junto a ellos se cbservaron elementos purpúricos puntiformes. Otra marifestación cutánea es la presencia de zonas tojizas en nappe que se ubican en ambas mejillas.

E1 estudio de nuestras observaciones nos permite establecer una notoria influencia de la droga sobre la temperatura. que se manifiesta por alza térmica. La curva febril puede adquirir varios tipos en relación con la modalidad de uso de la droga. Cuando se aplica diariamente, se observa una alzal de ia temperatura en forma continua, que desciende cuando s: dicminuye la dosis intratecal $10 b s . \mathrm{N}^{\circ} 2 \mathrm{i}$. A este respecto 2.5 de interés consignar el hecho que, no obstante la desaparición de los signes clínicos de la meringitis y la evidente mejoria de los caracteres del líquido céfalc raquideo, se mantiene durante muchos días una remperatura sub febril de carácter re mitente que, a nuestro juicio, sería también condicionada po: la droga. porque hemos observado su desaparición varios días después de haber suspendido todo 1ratamiento.

\section{Comentario.}

En los dos casos de meningitis tuberculosa, cuyas historias clinicas hemos expuesto en esta comunicación. la estreptomicina ha tenide una eficacia que podemcs conceptuar, sin temor de incurrir en una exageración, de sorptendente en el sentido de provocar la regresión rápida de la sintomatología 
clínica. Esta eficacia extraordinatia se ha puesto más claramente en evidencia en la niñita de 13 años que cortesponde a la Obs. $N^{\circ}$ l. en la cual, las dosis masivas de estreptomicina. en un principio exclusivamente intraraquídea, lograron recuperarla del coma meníngeo con fenómenos graves de compromiso bulbar. A la fecha han transcurrido 7 meses desde la iniciación de su tratamiento.

Ha tenido dos recidivas cuya sintomatolegía ba consistido en ligera cefalea. temperatura ocasional durante el día, desmejoria del líquido céfalo raquídeo, que se ha manifestado por disminución de la glucosa y cloruros y aumento de las prcteínas y elementes celulares. Ha mantenido siempre un espléndido estado subjetivo y no se ha observado la intercarrencia de ningún otro signo meníngeo. Reiniciado el tratamiento en forma combinada, intramuscular e intraraquide.. se ha observado la remisión de los sintomas antes descritos.

En el ctro enfermito de 2 años (Obs. No 2), el tratamiento se ha iniçado con sintomas meningeos plenamente establecidos $a$ incluso con obnubilación sensorial. Ambes pacientes muestran en la radiografía pulmonar un complejo primario tuberculeso en evolución.

En el enfermito de la Obs. No 2. después de haber dimostrado una mejoría estable. tanto clínica como del líquido céfalo raquideo, se suspendió el tratamiento durante un mes. en cuyo lapso se han practicado semanalmente punciones di contrcl. Durante este perícdo el líquido céfalo raquideo ha experimentado una mejoría espontánea. hasta llegar casi a la normalidad. Esta tendencia a la normalización la hemos observado en los dos pacientés que han tenido períodos más" menos prolongadcs de descanso. Es por esta razón que. a nuestro juicio. no se puede formar un concepto exacto sobre la mejoría que ha experimentado el líquido céfalo raquideo durante el tratamiento. pues ésta se hace evidente en los periodos de descanso. durante los cuales ha dejado de ejercer la droga un efecto, al parecer. irritativo sobre las meninges.

En les dos casos. e íncluso una tercera observación que no se incluye en esta comunicación por ser de muy reciente data, la mejoría clínica se ha hecho evidente más o menos al $6^{\circ}$ día de tratamiento.

Si consideramos en conjunto el estado actual de recuperación física $y$ subjetiva de los pacientes con meningitis tuberculosa que presentamos, podemos decir que da la impresión de niños que están en condiciones normales de salud. excepto la persistencia de alteraciones en el líquido céfalo ra- 
quídeo. Este es el motivo por el cual no podemos aún hablar de mejcría definitiva $y$, en consecuencia, debemos mostrar siempre una actitud de reserva ante la posible contingencia de nuevas reagudizaciones de la enfermedad.

Como consecuencia lógica de lo antes expuesto, cabe formular las preguntas siguientes: ¿Es posible llegar a obtener una mejoría completa y definitiva de la meningitis tuberculosa mediante el uso de la estreptomicina? ¿Cuánto tiempo requiere cl tratamiento?

Debido a la escasa experiencia universal que aun se tiene respecto a los resultados de la estreptomicina en la meningitis tuberculcsas y los casos relatados en la literatura extranjera, no tienen todavía un período de observación suficientemente largo, más cuando en uno de ellos se ha observado reactivación del proceso meníngeo (Hinshaw y Feldman), la recuperación completa y definitiva de la mieningitis tuberculosa constituye, hoy en día. una interrogante que perdurará hasta que los ensayos que se practican en la actualidad, nos muestren en un futuro próximo, casos cuya curación sea indiscutible.

Un hecho que parece evidente, a juzgar por el periodo tan prolongado que requiere el tratamiento para obtener und mejería clínica satisfactoria, como asimismo la reagudización del proceso meníngeo en el caso ya citado y en la Obs. $N^{\circ} 1$ de nuestra comunicación es que, si bien la droga produce una regresión rápida de los signos meníngeos y subjetivos del paciente, el proceso permanece en un estado de actividad latente, detido, pcsiblemente, a una inhibición del crecimiento y atenuación de la virulencia del bacilo de Koch; pero se obsersa que durante los períodes de descanso es susceptible de adquirir nuevamente su actividad.

Consecuente con lo que acabamos de exponer, y siguiendo la técrica sobre la cual hemes becho referencia, el tratamiento debe ser mantanido por un tiempo prolongado. Durante los pericdos de descanso es necesario asumir una actitud de expectación, para reiniciarlo inmediatamente ante cualquier indicic de reagudización, revelade tante por los signos clínicos como por el examen del liquido céfalo raquídeo, cuyo contrcl debe practicarse periódicamente.

En nuestro concepto, tanto más cuando existen lesiones cbjetivas a la pantalla de compromiso pulmonar, el tratamiento debe ser necesariamente mantenido por un minimo de 10 a 12 meses. o sea, durante un lapso al final del cual habitualmente se observa la calcificación del proceso pulmonar de la primo infección tuberculosa. 


\section{Sumario.}

$1^{\circ}$ Dos pacientes con meningitis tuberculosa, probada bacteriológicamente, fueron tratados con. estreptomicina.

$2^{\circ}$ La cantidad de droga usada ha sido la más alta hasta la fecha. Se usó la via intramuscular e intraraquídea.

$3^{\circ}$ El niño de 22 meses recibió 50 gramos en los 2 primeros meses de tratamiento. La niña de 13 años recibió 136 gramos en los primeros 5 meses.

$4^{\circ}$ Ambos pacientes presentan un foco activo $d_{\ell}$ tuberculosis pulmonar. Hemos observado que con esta terapia se observa mejoria ya en la primera semana. Nuestros pacientis, después de 4 y 7 meses de tratamiento, están clínicamente bien.

$5^{\circ}$ Junto al tratamiento y como consecuencia de él. observamos: dolores radiculares; convulsiones: estralbismo transitorio; exageración de los signos meningeos y gran prurito.

$6^{\circ}$ Cremos que la terapia con estreptomicina debe mantenerse mientras existan focos tuberculosos activos.

\section{Summary.}

1) Two patients with bacteriologically proved TBC meningitis have been treated with streptomycin.

2) As far as we know the drug dosage has been the largest one used in this condition. The combined intramuscular and intrathecal routes were used.

3) A total amount of alfost $50 \mathrm{Gm}$. of streptomycin wer: administered to a 22 months old boy in the first two months of treatment. A thirteen years old girl received over $136 \mathrm{Gm}$. on the first five months with intermittent rest periods.

4) Both patients also show an active focus of pulmonary tuberculose. So far, we have obstrved a definite improvement with this type of therapy which was evident even on the first week and now. after four and seven months of treatment our two cases ate clinically well.

5) Along with treatment we observed sides effects of the drug consisting on: radicular pain; convulsions; transient estrabismus; exaggeration of the meningeals signs and a marked prurito.

6) We think the streptomycin therapy should be maintain as long as there is any active TB focus. In our cases the lungs. 


\section{Bibliografia.}

1) COOKE. DUNPHY and BI.AKE. - Mencionados en la publicación del National Rescarch Council. J. A. 'M. A. Vol. 132, pág. 4. 1946 ..

2. NATIONAI, RESEARCH COUNCIL. - Streptomycin in infections. The J. A. M. A. Vol, 132, pág. 4. 1946.

3) NATIONAL RESEARCH COUNCIL. - Streptomycin in infections. The J. A. M. A. Vol. 132, pág. 70. 1946.

4) FINLAND MAXVELL and MURRAY. - Streptomycin resistance. The J. A. M. A. Vol. 132, pág. 16. 1946.

5) NICHOL R., DONALD and HERREL, E. W. - Streptomycin. The J. A. M. A. Vot. 132, pág. 200. 1946.

6) HINSHAW g FELDMAN. - Treatment of Tuberculose with Streptomycin. The J. A. M. A. Vol, 132. pág. 778. 1946

7) OTTEMBERG, DONALD and BONDI, AMADEO. - Streptomycin Therapy. The J. A. M. A. Vol. 132, pag. 634. 1946..

8) KRAFCHICK L., LOUIS, - Tuberculose Meningitis. The J. A. M. A. Vol, 132, pàg. 375. 1946.

9) HEWITT, L. W. and CURRY, D. J. - Pharmacodynamic effect in man of Streptompcin containing a histamine like factor. The Journal of Lab. and Clin. Medicine. Vol, 32, pág. 42. 1947. 